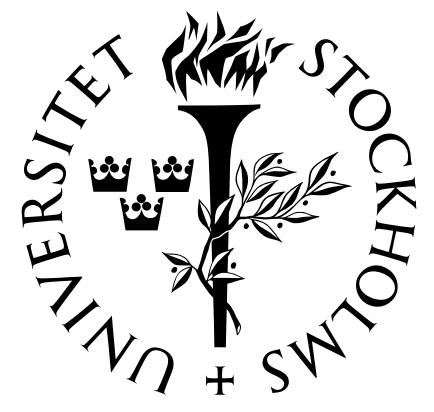

\title{
Spectral Gap for Complete Graphs: \\ Upper and Lower Estimates
}

Pavel Kurasov

Research Reports in Mathematics

Number 2, 2015

Department of Mathematics

STOCKHOLM UNIVERSITY 
Electronic version of this document is available at http://www.math.su.se/reports/2015/2

Date of publication: January 10, 2015.

2010 Mathematics Subject Classification:

Primary 34L25, 81U40; Secondary 35P25, 81V99.

Keywords: Quantum graphs, spectral gap.

Postal address:

Department of Mathematics

Stockholm University

S-106 91 Stockholm

Sweden

Electronic addresses:

http://www.math.su.se/

info@math.su.se 


\title{
SPECTRAL GAP FOR COMPLETE GRAPHS: UPPER AND LOWER ESTIMATES
}

\author{
P. KURASOV
}

\begin{abstract}
Lower and upper estimates for the spectral of the Laplacian on a compact metric graph are discussed. New upper estimates are presented and existing lower estimates are reviewed. The accuracy of these estimates is checked in the case of complete (not necessarily regular) graph with large number of vertices.
\end{abstract}

\section{INTRODUCTION}

Let $\Gamma$ be a compact connected metric graph formed by a finite number of compact edges $E_{n}, n=1,2, \ldots, N$. Let us denote by $L(\Gamma)$ the corresponding Laplace operator defined on $W_{2}^{2}$-functions $u$ satisfying standard matching/boundary conditions at every vertex:

$$
\left\{\begin{array}{l}
u \text { is continuous, } \\
\text { the sum of normal derivatives is equal to zero. }
\end{array}\right.
$$

The operator $L(\Gamma)$ is self-adjoint and is completely determined by the metric graph $\Gamma$. The spectrum is nonnegative and consists of an infinite sequence of eigenvalues $\lambda_{j}$ of finite multiplicity tending to $+\infty$. The lowest eigenvalue (the ground state) is zero $\lambda_{0}=0$ and the corresponding eigenfunction is just a constant function on $\Gamma$. The multiplicity of $\lambda_{0}$ is one, since the graph is connected. Our main interest here is the distance between the first two eigenvalues to be called the spectral gap. Since $\lambda_{0}=0$ the spectral gap coincides with $\lambda_{1}$. For general introduction into the theory differential operators on metric graphs see $[2,6,11]$.

The spectral gap is not only an important parameter determining asymptotic behavior of solutions to non-stationary Schrödinger equation, but is closely related to connectivity properties of graphs (see [1] for a generalization of Cheeger's constant for discrete graphs [3]).

The aim of the current paper is to discuss lower and upper estimates for the spectral gap for the Laplacian on a metric graph. We review existing lower estimates and obtain new effective upper estimates. Applicability of these estimates is checked in the case of complete graphs with large number of vertices. Note that it is not assumed that the graphs are regular, i.e. all edges have the same length. In particular obtained estimates allow to prove that the spectral gap remains open in the limit of large number of vertices, provided the lengths of the edges remain separated from zero.

The author was supported in part by Swedish Research Council Grant \#50092501. 


\section{LOWER ESTIMATES}

The following lower estimate for the spectral gap has been proven in [4] and [5]

$$
\frac{\pi^{2}}{\mathcal{L}^{2}} \leq \lambda_{1}(\Gamma)
$$

This estimate can be improved by factor 4 if the graph $\Gamma$ is of even degree (i.e. all vertices have even degree)

$$
4 \frac{\pi^{2}}{\mathcal{L}^{2}} \leq \lambda_{1}(\Gamma)
$$

Cheeger's ideas allow to prove another one lower estimate $[9,10]$

$$
\frac{h(\Gamma)^{2}}{4} \leq \lambda_{1}(\Gamma)
$$

where the Cheeger constant is defined as

$$
h(\Gamma)=\inf _{P} \frac{|P|}{\min \left\{\mathcal{L}\left(\Gamma_{1}\right), \mathcal{L}\left(\Gamma_{2}\right)\right\}},
$$

where the minimum is taken over all cuts $P$ of $\Gamma$ dividing the graph into two components $\Gamma_{1}$ and $\Gamma_{2} .|P|$ denotes the number of points in $P$. This formula is obtained using co-area formula is a direct generalization of the classical Cheeger's formula obtained for Laplacian on Riemannian manifolds [3].

\section{UpPer estimate}

3.1. The first estimate. We follow here approach suggested in [7]. Any eigenfunction corresponding to the first excited eigenvalue $\lambda_{1}(\Gamma)$ should attain both positive and negative values. Therefore one may obtain a surprisingly effective estimate by cutting the graph $\Gamma$ into two disjoint components. One can think about these components as certain models for the nodal domains for the first eigenfunction, also in general they do not coincide. The estimate we are going to prove is valid for arbitrary even non-complete metric graphs $\Gamma$.

The idea is to cut of several edges in $\Gamma$, so that the remaining graph graph is not connected anymore. Let us denote the edges to be deleted by $E_{n_{j}}$ and introduce the set $S=\cup_{j=1}^{s} E_{n_{j}}$. If the resulting graph $\Gamma \backslash S$ is not connected, then we say that $S$ is a proper cut of $\Gamma$. The set $\Gamma \backslash S$ may consist of several connected components. Let us denote by $\Gamma_{1}$ and $\Gamma_{2}$ any separation of $\Gamma \backslash S$ into two nonintersecting sets

$$
\Gamma_{1} \cup \Gamma_{2}=\Gamma \backslash S, \quad \Gamma_{1} \cap \Gamma_{2}=\emptyset .
$$

We assume in this section that $\Gamma$ contains no loops, i.e. edges adjusted to one vertex. This is not an important restriction. Really, consider any graphs $\Gamma$ with a loop, mark any point on the loop and put a new vertex at this point. The new metric graph obtained in this way contains no loops but the corresponding Laplace operator is unitary equivalent to the Laplace operator on the original graph.

With any set $S$ as described above let us associate the Cheeger quotient

$$
c_{S}(\Gamma)=\min _{\Gamma_{1}, \Gamma_{2}: \quad \begin{array}{l}
\Gamma_{1} \cup \Gamma_{2}=\Gamma \backslash S ; \\
\Gamma_{1} \cap \Gamma_{2}=\emptyset
\end{array}} \frac{\mathcal{L}(\Gamma) \sum_{E_{n} \subset S} \ell_{n}^{-1}}{\mathcal{L}\left(\Gamma_{1}\right) \mathcal{L}\left(\Gamma_{2}\right)},
$$


Consider the function $g$ defined as follows

$$
g(x)= \begin{cases}1, & x \in \Gamma_{1} \\ -1, & x \in \Gamma_{2} \\ \ell_{n}^{-1}\left(-\operatorname{dist}\left(x, \Gamma_{1}\right)+\operatorname{dist}\left(x, \Gamma_{2}\right)\right), & x \in E_{n} \subset S,\end{cases}
$$

where the distances dist $\left(x, \Gamma_{j}\right), j=1,2$ are calculated along the corresponding interval $x \in E_{n}$. The continuous function $g$ is constructed in such a way, that it is equal to \pm 1 on $\Gamma_{1}$ and $\Gamma_{2}$ and is linear on the edges connecting $\Gamma_{1}$ and $\Gamma_{2}$. But the mean value of the function might be different from zero. In that case the function $g$ has to be modified so that it will be orthogonal to the ground state. Consider then the function $f$ which is not only continuous, but also orthogonal to the ground state:

$$
f(x)=g(x)-\mathcal{L}(\Gamma)^{-1}\langle g, 1\rangle_{L_{2}(\Gamma)} .
$$

The Rayleigh quotient for the function $f$ gives an upper estimate for the spectral gap:

$$
\lambda_{1}(\Gamma) \leq \frac{\int_{\Gamma}\left|f^{\prime}(x)\right|^{2} d x}{\int_{\Gamma}|f(x)|^{2} d x} .
$$

Let us calculate the Dirichlet integral and the norm of $f$ :

$$
\begin{aligned}
\left\|f^{\prime}\right\|_{L_{2}(\Gamma)}^{2} & =\left\|g^{\prime}\right\|_{L_{2}(\Gamma)}^{2}=\sum_{E_{n} \subset S} \int_{E_{n}}\left(-2 \ell_{n}^{-1}\right)^{2} d x=4 \sum_{E_{n} \subset S} \ell_{n}^{-1} ; \\
\|f\|_{L_{2}(\Gamma)}^{2} & =\|g\|_{L_{2}(\Gamma)}^{2}-\mathcal{L}(\Gamma)^{-1}\langle g, 1\rangle^{2} \\
& \geq \mathcal{L}\left(\Gamma_{1}\right)+\mathcal{L}\left(\Gamma_{2}\right)-\mathcal{L}(\Gamma)^{-1}\left(\mathcal{L}\left(\Gamma_{1}\right)-\mathcal{L}\left(\Gamma_{2}\right)\right)^{2} \\
& \geq 4 \frac{\mathcal{L}\left(\Gamma_{1}\right) \mathcal{L}\left(\Gamma_{2}\right)}{\mathcal{L}(\Gamma)} .
\end{aligned}
$$

This gives the following upper estimate for $\lambda_{1}(\Gamma)$

$$
\lambda_{1}(\Gamma) \leq c_{S}(\Gamma)
$$

where we use (6). We have proven the following

Theorem 1. Let $\Gamma$ be a connected metric graph without loops, then the spectral gap is estimated from above by Cheeger's constant

$$
\lambda_{1}(\Gamma) \leq C(\Gamma):=\min _{S-\text { proper cut of } \Gamma \quad \Gamma_{1}, \Gamma_{2}:} \begin{aligned}
& \min _{1} \cup \Gamma_{2}=\Gamma \backslash S \\
& \Gamma_{1} \cap \Gamma_{2}=\emptyset
\end{aligned} \quad \begin{aligned}
& \frac{\mathcal{L}(\Gamma) \sum_{E_{n} \subset S} \ell_{n}^{-1}}{\mathcal{L}\left(\Gamma_{1}\right) \mathcal{L}\left(\Gamma_{2}\right)} \\
&
\end{aligned}
$$

Proof. The result follows immediately from estimate (10) taking into account that the set $S$ dividing $\Gamma$ into disconnected components is arbitrary.

Note that the subgraphs used in the latter theorem does not necessarily coincide with the nodal domains for the first eigenfunction. Moreover the function $f$ introduced above is not an eigenfunction corresponding to $\lambda_{1}(\Gamma)$. Nevertheless this function substituted into the Rayleigh quotient gives a good upper approximation for the spectral gap. 
3.2. The second (improved) estimate. Our choice of the function $g$ above was rather ruff and therefore the equality in the obtained estimate is never achieved. One may improve the estimate by considering a more realistic candidate for the first eigenfunction. Let us choose the function $g$ as follows (instead of (7)):

$$
g(x)= \begin{cases}1, & x \in \Gamma_{1} ; \\ \cos \frac{\operatorname{dist}\left(x, \Gamma_{1}\right)}{\ell_{n}} \pi=-\cos \frac{\operatorname{dist}\left(x, \Gamma_{2}\right)}{\ell_{n}} \pi, & x \in E_{n} \subset S ; \\ -1, & x \in \Gamma_{2} .\end{cases}
$$

We again shift the function by a constant:

$$
f(x)=g(x)-\frac{\mathcal{L}\left(\Gamma_{1}\right)-\mathcal{L}\left(\Gamma_{2}\right)}{\mathcal{L}(\Gamma)} .
$$

and calculate explicitly the norms appearing in the Rayleigh quotient

$$
\begin{gathered}
\left\|f^{\prime}\right\|_{L_{2}(\Gamma)}^{2}=\sum_{E_{n} \subset S}\left(\frac{\pi}{\ell_{n}}\right)^{2} \int_{E_{n}} \sin ^{2} \frac{\operatorname{dist}\left(x, \Gamma_{1}\right)}{\ell_{n}} \pi d x \\
=\frac{\pi^{2}}{2} \sum_{E_{n} \subset S} \ell_{n}^{-1} \\
\|f\|_{L_{2}(\Gamma)}^{2}=\|g\|_{L_{2}(\Gamma)}^{2}-\frac{\left(\mathcal{L}\left(\Gamma_{1}\right)-\mathcal{L}\left(\Gamma_{2}\right)\right)^{2}}{\mathcal{L}(\Gamma)} \\
\|g\|_{L_{2}(\Gamma)}^{2}=\mathcal{L}\left(\Gamma_{1}\right)+\mathcal{L}\left(\Gamma_{2}\right)+\frac{1}{2} \mathcal{L}(S) .
\end{gathered}
$$

The Rayleigh quotient gives the following estimate for the second eigenvalue

$$
\begin{aligned}
\lambda_{1}(\Gamma) & \leq \frac{\left\|f^{\prime}\right\|_{L_{2}(\Gamma)}^{2}}{\|f\|_{L_{2}(\Gamma)}^{2}} \\
& =\frac{\frac{\pi^{2}}{2} \sum_{E_{n} \subset S} \ell_{n}^{-1}}{\mathcal{L}\left(\Gamma_{1}\right)+\mathcal{L}\left(\Gamma_{2}\right)+\frac{1}{2} \mathcal{L}(S)-\frac{\left(\mathcal{L}\left(\Gamma_{1}\right)-\mathcal{L}\left(\Gamma_{2}\right)\right)^{2}}{\mathcal{L}(\Gamma)}} \\
& =\frac{\pi^{2} \mathcal{L}(\Gamma) \sum_{E_{n} \subset S} \ell_{n}^{-1}}{8 \mathcal{L}\left(\Gamma_{1}\right) \mathcal{L}\left(\Gamma_{2}\right)+3\left(\mathcal{L}\left(\Gamma_{1}\right)+\mathcal{L}\left(\Gamma_{2}\right)\right) \mathcal{L}(S)+\mathcal{L}^{2}(S)} .
\end{aligned}
$$

The new obtained estimate is not as explicit as the estimate (11), but its advantage is that the equality is attained if the graph $\Gamma$ is a loop and the subgraphs $\Gamma_{1}$ and $\Gamma_{2}$ are given by two opposite points on the loop.

3.3. The third (universal) estimate. Let us use the same idea to get an upper estimate, which does not require considering proper cuts, since it is not clear which proper cut gives the best estimate.

We shall need the following definition: $A$ graph $\Gamma$ is called bipartite if the vertices can be divided into two sets $\mathbb{V}_{1}$ and $\mathbb{V}_{2}$, so that the edges connect only vertices from different sets. In other words the vertices in $\Gamma$ can be colored by two colors, so that neighboring vertices have different colors.

Theorem 2. The spectral gap for the Laplace operator on a metric graph $\Gamma$ satisfies the following upper estimates

$$
\lambda_{1}(\Gamma) \leq \frac{\pi^{2}}{\mathcal{L}(\Gamma)} 4 \sum_{E_{n} \subset \Gamma} \ell_{n}^{-1} .
$$


If the metric graph $\Gamma$ is bipartite, then the upper estimate can be improved by the factor 4 as follows

$$
\lambda_{1}(\Gamma) \leq \frac{\pi^{2}}{\mathcal{L}(\Gamma)} \sum_{E_{n} \subset \Gamma} \ell_{n}^{-1} .
$$

Proof. Let us start from the second formula. Assume that the graph $\Gamma$ is bipartite. Then the graphs $\Gamma_{1}$ and $\Gamma_{2}$ appearing in Cheeger's estimate (16) can be chosen equal to vertex sets $\mathbb{V}_{1}$ and $\mathbb{V}_{2}$ from the definition of a bipartite graph. The proper cut set $S$ contains all edges. In other words we cut all edges in the graph $\Gamma$. Since $\mathcal{L}\left(\Gamma_{1}\right)=\mathcal{L}\left(\Gamma_{2}\right)=0$ and $\mathcal{L}(S)=\mathcal{L}(\Gamma)$ we get the following estimate

$$
\lambda_{1}(\Gamma) \leq \frac{\pi^{2}}{\mathcal{L}(\Gamma)} \sum_{E_{n} \subset \Gamma} \ell_{n}^{-1} .
$$

The upper estimate for arbitrary graphs can be proven by the following trick: any metric graph $\Gamma$ can be turned into a bipartite graph by introducing new vertices in the middle of every edge. Then the sets $\mathbb{V}_{1}$ and $\mathbb{V}_{2}$ can be chosen equal to the unions of old and new vertices respectively. Then already proven estimate gives

$$
\lambda_{1}(\Gamma) \leq \frac{\pi^{2}}{\mathcal{L}(\Gamma)} 2 \sum_{E_{n} \subset \Gamma}\left(\ell_{n} / 2\right)^{-1} .
$$

Factor 2 in front of the sum appears due to the fact that every edge in $\Gamma$ is divided into two smaller edges of lengths $\ell / 2$.

\section{Complete Graphs: How Good obtained estimates ARE?}

In what follows we are going to discuss how good obtained estimates work for the complete (not necessarily regular) graphs.

The aim of this note is to provide effective estimates for the spectral gap of a complete graph assuming that the lengths of edges may be different. We would like to exclude graphs with very short and very long edges and therefore assume that the lengths $\ell_{n}$ of all edges satisfy the following

\section{Assumption 1.}

$$
0<\ell_{\min } \leq \ell_{n} \leq \ell_{\max }<\infty .
$$

Note that only complete graphs will be considered in the rest of this note. The number of vertices will be denoted by $M$, so the number of edges is $N=M(M-$ $1) / 2$.

4.1. Regular complete graphs: formula for the spectral gap. Let us consider first the regular complete graph $K_{M}$, i.e. a complete graph on $M$ vertices with all edges having the same length to be denoted by $\ell$. Then the total length of the graph is

$$
\mathcal{L}=\frac{M(M-1)}{2} \ell .
$$

Our immediate goal is to calculate the spectrum of the corresponding Laplacian $L\left(K_{M}\right)$. 


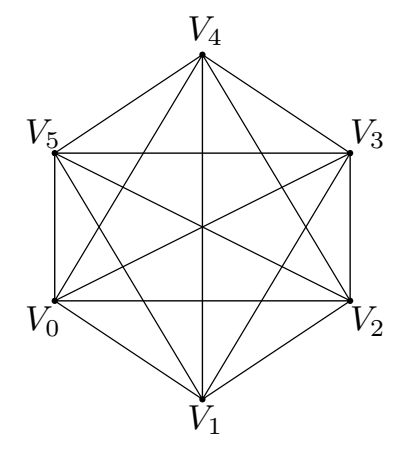

Figure 1. Complete graph $K_{6}$.

The vertices in the graph can be numbered as $V_{j}, j=0,1,2, \ldots, M-1$. Consider the rotation operator $R: V_{j} \mapsto V_{j+1}$. We keep the same notation for the induced map between the edges. It is clear that $R^{M}=I$ and $R L\left(K_{M}\right)=L\left(K_{M}\right) R$, hence the eigenfunctions $\psi$ for the Laplacian can be constructed being quasi invariant

$$
R \psi_{z}=z^{-m} \psi_{z}, \quad z=e^{i \frac{2 \pi}{M}}, m=0,1,2, \ldots, M-1 .
$$

It is then clear that every such function $\psi$ is completely determined by its values on the edges attached to one of the vertices, say $V_{0}$. These edges will be called the fundamental domain in what follows. 


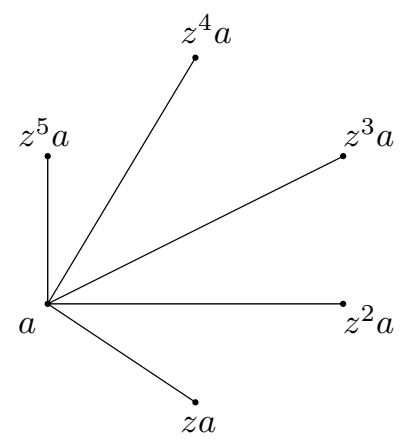

Figure 2. The fundamental domain for $\psi_{z}$. Values of the function are marked.

Let us denote by $a$ the value of the function $\psi_{z}$ at the zero vertex: $\psi_{z}\left(V_{0}\right)=$ $a$. It follows that $\psi_{z}\left(V_{j}\right)=z^{j} a$. It will be convenient to identify every edge $E_{j}$ connecting $V_{0}$ and $V_{j}$ with its own copy of the interval [0, $\ell$ ], where $x=0$ corresponds to $V_{0}$. Then the function $\psi_{z}$ can be reconstructed on all edges belonging to the fundamental domain

$$
\psi_{z}(x)=-a \frac{\sin k(x-\ell)}{\sin k \ell}+z^{j} a \frac{\sin k x}{\sin k \ell}, \quad x \in E_{j}, \quad k \neq \frac{n \pi}{\ell}, n \in \mathbb{N} .
$$

The function constructed in this way is continuous at $V_{0}$ and it remains to check that the condition on the sum of normal derivatives is satisfied

$$
\sum_{j=1}^{M-1}\left(-a k \frac{\cos k \ell}{\sin k \ell}+z^{j} a k \frac{1}{\sin k \ell}\right)=0 .
$$

Consider first the case $z=1$. Equation (20) implies

$$
\cos k \ell-1=0 \Rightarrow k=0, \frac{2 \pi}{\ell}, \frac{4 \pi}{\ell}, \ldots
$$

$k=0$ corresponds to the ground state constant eigenfunction, which is invariant under the rotation $R$.

For $z \neq 1$ (20) implies that

$$
(M-1) \cos k \ell+1=0,
$$

where we used that $z+z^{2}+\ldots z^{M-1}=-1$. It follows that the eigenvalue $\lambda_{1}\left(K_{M}\right)$ has multiplicity $M-1$ and is given by

$$
k_{1}\left(K_{M}\right)=\frac{1}{\ell} \arccos \left(-\frac{1}{M-1}\right) .
$$


Here we took into account that the smallest nonzero $k$ solving $\sin k \ell=0$ is $\pi / \ell$ and it is always greater than the calculated $k_{1}\left(K_{M}\right)$.

Hence we have shown that for the complete regular graph $K_{M}$ the spectral gap is given by

$$
\lambda_{1}\left(K_{M}\right)=\frac{M^{2}(M-1)^{2}}{4 \mathcal{L}^{2}}\left(\arccos \left(-\frac{1}{M-1}\right)\right)^{2} \underset{M \rightarrow \infty}{\sim} \frac{\pi^{2}}{16 \mathcal{L}^{2}} M^{4},
$$

and its value increases proportionally to $M^{4}$. Hence no universal upper estimate for the spectral gap for a graph of a fixed total length is available unless we restrict the number of vertices.

4.2. Lower estimates. Our aim is to prove that the spectral gap for complete graphs does not go to zero as the number of vertices tends to infinity, provided the lengths of the edges satisfy Assumption 1.

Consider first the universal estimate obtained by Friedlander-Kurasov-Naboko

$$
\lambda_{1} \geq \frac{\pi^{2}}{\mathcal{L}(\Gamma)^{2}} \geq \frac{\pi^{2}}{\ell_{\max }^{2}} \frac{4}{M^{2}(M-1)^{2}} \rightarrow_{M \rightarrow \infty} 0 .
$$

Hence this estimate is not good for our purposes, since it does not allow one to prove that the spectral gap remains closed in the limit $M \rightarrow \infty$.

Let us turn to Cheeger's estimate (3). The problem working with this estimate is that we do not know, which open subset $Y$ does minimize the quotient. But we may try to get a lower estimate for the Cheeger constant

$$
h:=\inf _{P} \frac{|P|}{\min _{i} \mathcal{L}\left(\Gamma_{i}\right)},
$$

where the infimum is taken over all edge-cuts $P$ of the graph $\Gamma$ dividing it into two parts denoted by $\Gamma_{1}$ and $\Gamma_{2}$. Here $\mathcal{L}\left(\Gamma_{i}\right)$ is the total length of $\Gamma_{i}$ and $|P|$ is the number of edge cuts.

Consider the complete graph $K_{M}$ with $M$ vertices. It is not easy to understand which particular cut minimizes the Cheeger quotient, especially since we do not know the precise values of the dodge lengths. But we may estimate this constant from below. Assume that the minimizing cut $P$ divides $K_{M}$ into two graphs $\Gamma_{1}$ and $\Gamma_{2}$ with $M_{1}$ and $M_{2}$ vertices respectively. (Note, we do not assume to know $M_{1}$.) Without loss of generality we require that $M_{1} \leq M_{2}$.

The number of cuts can easily be calculated

$$
|P|=\underbrace{\frac{M(M-1)}{2}}_{\text {number of edges in } \Gamma}-\underbrace{\frac{M_{1}\left(M_{1}-1\right)}{2}}_{\text {number of edges in } \Gamma_{1}}-\underbrace{\frac{\left.M_{2}\left(M_{2}-1\right)\right)}{2}}_{\text {number of edges in } \Gamma_{2}}
$$

We have assumed that $M_{1} \leq M_{2}$, therefore it is natural that $\mathcal{L}\left(\Gamma_{1}\right) \leq \mathcal{L}\left(\Gamma_{2}\right)$, but it does not really matter, since we get

$$
\begin{aligned}
\mathcal{L}\left(\Gamma_{1}\right) & \leq \ell_{\max }\{\underbrace{\frac{M_{1}\left(M_{1}-1\right)}{2}+\underbrace{|P|}_{\text {number of cutted edges }}}_{\text {number of edges in } \Gamma_{1}}\} \\
& =\ell_{\max }\left\{\frac{M(M-1)}{2}-\frac{\left.M_{2}\left(M_{2}-1\right)\right)}{2}\right\} .
\end{aligned}
$$


It follows that

$$
\begin{aligned}
h & \geq \frac{\left.M(M-1)-M_{1}\left(M_{1}-1\right)-M_{2}\left(M_{2}-1\right)\right)}{\left.\ell_{\max }\left\{M(M-1)-M_{2}\left(M_{2}-1\right)\right)\right\}} \\
& =\frac{1}{\ell_{\max }}\left\{1-\frac{M_{1}\left(M_{1}-1\right)}{M(M-1)-M_{2}\left(M_{2}-1\right)}\right\} \\
& =\frac{1}{\ell_{\max }}\left\{1-\frac{M_{1}\left(M_{1}-1\right)}{M_{1}\left(M_{1}-1\right)+2 M_{1} M_{2}}\right\} \\
& >\frac{1}{\ell_{\max }}\left\{1-\frac{M_{1}\left(M_{1}-1\right)}{M_{1}\left(M_{1}-1\right)+2 M_{1}\left(M_{1}-1\right)}\right\} \\
& =\frac{2}{3 \ell_{\max }} .
\end{aligned}
$$

With this we get the following estimate for the spectral gap:

$$
\lambda_{1} \geq \frac{1}{4} h^{2}>\frac{1}{9 \ell_{\max }^{2}} .
$$

Our conclusion: the spectral gap remain finite even for large complete graphs, provided Assumption 1 holds.

4.3. Upper estimates. We examine now the upper estimates obtained in Section3. We consider arbitrary not necessarily regular complete graph $K_{M}$ satisfying Assumption 1.

We start with the first estimate (11). Since we do not know precise values of the edge lengths, it is impossible to check which particular proper cut minimizers the upper estimate. But it is not really necessary, we may assume as before that the graphs $\Gamma_{1}$ and $\Gamma_{2}$ contain $M_{1}$ and $M_{2}$ vertices $\left(M_{1} \leq M_{2}\right)$.

Let us estimate the quantities appearing in (11):

$$
\begin{aligned}
\mathcal{L} & \leq \ell_{\max } M(M-1) / 2 ; \\
\sum_{E_{n} \subset S} \ell_{n}^{-1} & \leq \ell_{\min }^{-1}\left(M(M-1)-M_{1}\left(M_{1}-1\right)-M_{2}\left(M_{2}-1\right)\right) / 2 ; \\
\mathcal{L}\left(\Gamma_{j}\right) & \geq \ell_{\min } M_{j}\left(M_{j}-1\right) / 2, \quad j=1,2 .
\end{aligned}
$$

These estimates imply

$$
\lambda_{1}\left(K_{M}\right) \leq \frac{\ell_{\max }}{\ell_{\min }^{3}} \frac{M(M-1) 2 M_{1} M_{2}}{M_{1}\left(M_{1}-1\right) M_{2}\left(M_{2}-1\right)} .
$$

Since the numbers $M_{1}$ and $M_{2}$ are arbitrary subject to $M_{1}+M_{2}=M$ we have the following estimates

$$
\lambda_{1}\left(K_{M}\right) \leq 2 \frac{\ell_{\max }}{\ell_{\min }^{3}} M(M-1) \min _{M_{1}+M_{2}=M} \frac{1}{\left(M_{1}-1\right)\left(M_{2}-1\right)}
$$

and

$$
\lambda_{1}\left(K_{M}\right) \leq 2 \frac{\ell_{\max }}{\ell_{\min }^{3}} M(M-1)\left(\left[\frac{M}{2}\right]-1\right)^{-2} .
$$

In the limit of large $M$ we get

$$
\lambda_{1}\left(K_{M}\right) \leq 8 \frac{\ell_{\max }}{\ell_{\min }^{3}} .
$$

If the graph is in addition regular $\ell_{\max }=\ell_{\min }=\ell$ we have

$$
\lambda_{1}\left(K_{M}\right) \leq 8 \ell^{-2}, M \rightarrow \infty
$$


to be compared with the precise value

$$
\lambda_{1}\left(K_{M}\right) \sim \frac{\pi^{2}}{4} \ell^{-2} .
$$

We conclude that the estimate (11) predicts correct asymptotic behavior for the spectral gap of complete graphs, but the constants are of course not precise.

The second estimate gives approximately the same result, also calculations are not very explicit. Let us turn to the third estimate, which gives us

$$
\lambda_{1}\left(K_{M}\right) \leq \frac{\pi^{2}}{M(M-1) \ell_{\min }} 4 M(M-1) \ell_{\min }^{-1}=4 \pi^{2} \ell_{\min }^{-2} .
$$

If the graph is regular, then the estimate takes the form

$$
\lambda_{1}\left(K_{M}\right) \leq 4 \pi^{2} \ell^{-2}
$$

which is worse than (34), but the difference is not enormous.

Summing up obtained lower and upper estimates provide rather good approximations for the spectral gap for complete not necessarily regular graphs.

Acknowledgements. The author would like to thank Delio Mugnolo for organizing an extremely stimulating conference in Bielefeld. Many thanks go to Olaf Post for discussions concerning Cheeger estimate (4), which was proved by Rune Suhr [8]. The author is grateful to anonymous referee for correcting inaccuracies and fruitful remarks.

\section{REFERENCES}

[1] N. Alon, Eigenvalues and expanders, Combinatorica 6(1986), 8396.

[2] G. Berkolaiko and P. Kuchment, Introduction to Quantum Graphs, AMS 2013.

[3] J. Cheeger, A lower bound for the smallest eigenvalue of the Laplacian, in Problems in Analysis (edited by R. C. Gunning), Princeton University Press, New Jersey, (1970), 195-199.

[4] L. Friedlander, Extremal properties of eigenvalues for a metric graph., Ann. Inst. Fourier (Grenoble), 55 (2005), no. 1, 199211.

[5] P. Kurasov and S. Naboko, Rayleigh estimates for differential operators on graphs, J.Spectral Theory, 4 (2014), 211-219.

[6] P. Kurasov, Quantum graphs: spectral theory and inverse problems (in Print).

[7] P. Kurasov, On the spectral gap for Laplacians on Metric graphs, Acta Physica Polonica A, 124 (2013), 1060-1062.

[8] P. Kurasov, R. Suhr, Cheeger estimates for quantum graphs (in preparation).

[9] S. Nicaise, Spectre des réseaux topologiques finis. (French) [The spectrum of finite topological networks] Bull. Sci. Math. (2) 111 (1987), no. 4, 401413.

[10] O. Post, Spectral analysis of metric graphs and related spaces. Limits of graphs in group theory and computer science, 109140, EPFL Press, Lausanne, 2009.

[11] O. Post, Spectral analysis on graph-like spaces, Lecture Notes in Mathematics, 2039. Springer, Heidelberg, 2012.

Dept. of Math., Stockholm Univ., 10691 Stockholm, Sweden

E-mail address: kurasov@math.su.se 